\title{
3rd Multimodality Cardiovascular Molecular Imaging Symposium-Poster Abstracts
}

This two-day symposium from SNM will bring together individuals from multiple scientific disciplines-including chemistry, engineering, physics, molecular biology, cardiovascular physiology, and imaging sciences - with the goal of promoting the emerging field of cardiovascular molecular imaging. This meeting continues the work of previous conferences held at NIH.

The 2012 meeting is designed to continue this momentum and stimulate further growth in the field by attracting individuals from both the basic science and clinical communities, with a special emphasis on encouraging participation by junior scientists. The speaker roster includes experts in the field. The program will focus on advances in targeted imaging of the cardiovascular system including imaging of cardiovascular receptors, stem cell therapy, vascular biology, myocardial metabolism, and other relevant biological processes. The size of the meeting will facilitate interaction among speakers and registrants and foster collaboration among participants to stimulate further interest in this field. The abstracts presented here represent some of the most interesting ideas in molecular imaging research as applied to the cardiovascular system. The abstracts are listed alphabetically by first author, and those that were selected to receive the Young Investigator Travel Award are indicated by a footnote.

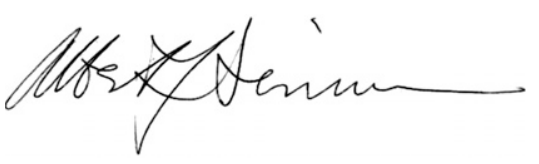

Albert J. Sinusas, MD

Director, Yale Translational Research Imaging Center (Y-TRIC) Yale University School of Medicine

\section{Abstract Reviewers}

Zahi A. Fayad, PhD

Mount Sinai School of Medicine, New York, NY

Ahmed Tawakol, MD

Massachusetts General Hospital, Boston, MA

Mehran M. Sadeghi, MD

Yale University School of Medicine, New Haven, CT

Lynne L. Johnson, MD

New York-Presbyterian Hospital/Columbia University Medical

Center, New York, NY
Joseph C. Wu, MD, PhD

Stanford University School of Medicine, Stanford, CA

Christopher M. Kramer, MD

University of Virginia, Charlottesville, VA

Albert J. Sinusas, MD

Yale University School of Medicine, New Haven, CT

Robert J. Gropler, MD

Washington University School of Medicine, St. Louis, MO 
Assessment of coronary artery wall remodeling using phase-sensitive dual inversion recovery MRI. K.Z. Abd-Elmoniem ${ }^{1}$, H. Zahiri ${ }^{2}$, R.I. Pettigrew $^{1}$, A.M. Gharib ${ }^{1} ;{ }^{1} \mathrm{NIH}$, NIDDK, Bethesda, MD, ${ }^{2} \mathrm{NIH}, \mathrm{CC}$, Bethesda, MD

Coronary arterial wall imaging is a promising noninvasive tool for quantitative assessment of positive arterial remodeling. Conventional techniques mandate imaging during cardiac rest period as well as nulling the blood signal inside the arterial lumen in order to generate an appropriate lumen-wall contrast. These contradicting requirements have limited coronary wall imaging to clinical research. Recently, phase-sensitive dual inversion recovery (PS-DIR) imaging (1) was proposed to alleviate the burden of nulling the blood signal and permitted more timing flexibility in planning for coronary wall imaging. This work investigates the utilization of PS-DIR in the assessment of coronary artery thickening in patients with coronary artery diseases (CAD). Methods: 23 subjects with calcified and non-calcified plaque, confirmed with CT angiography (CTA), were recruited for MRI coronary wall thickness assessment. For comparison, 23 normal subjects without known CAD or CAD risk factors were self-referred for the same MRI examination. A detailed assessment of CAD risk was performed and Framingham Risk Score was calculated. The location of imaging was chosen such that there was no visible plaque detected at the CTA. Anatomical slices perpendicular to the region of interest at endsystole were planned. A series of single-slice PS-DIR images was acquired with a fixed imaging time $(\mathrm{TI})=200 \mathrm{~ms}$ after the DIR pulse (slice thickness $=$ $8 \mathrm{~mm}, \mathrm{FOV}=190 \times 190 \mathrm{~mm}$, matrix $=320 \times 320$, interleaves $=20$, acquisition window $=18 \mathrm{~ms} /$ interleaf). Data were acquired during free breathing using 3-mm navigator gating and correction. The navigator was localized at the lung-liver interface of the right hemidiaphragm. Image quality was rated on the MR images using a 5-point scale. An image quality of 4 or above was considered adequate. Vessel SNR, CNR, and sharpness were measured on the signed-magnitude PS-DIR images. Mean vessel wall thickness was measured automatically on the images. Unpaired t-test was utilized to compare between normal and patient measurements. Results: Adequate image quality was seen in $76 \%$ of subjects. There was no difference in image CNR between the groups. At the locations without noticeable plaques, coronary wall thickness was thicker in patients in comparison to normal controls $(1.67 \mathrm{~mm}$ vs. $1.24 \mathrm{~mm}, \mathrm{p}<0.001)$ Conclusion: This study demonstrates that CAD patients with confirmed plaques have thicker wall thickness at sites without noticeable plaques. Reference: 1. Abd-Elmoniem KZ et al. Magn Res Med. 2010;63:1021-1030.

\section{2}

Monitoring inflammatory flux in canine myocardial infarction with 18-F FDG-PET. K.J. Blackwood ${ }^{1}$, M. Huegin ${ }^{2}$, L. Deans ${ }^{2}$, J. Sykes ${ }^{2}$, H. Marshall ${ }^{1}$, G. Wisenberg ${ }^{3}$, F.S. Prato ${ }^{4}$; ${ }^{1}$ Department of Medical Biophysics, University of Western Ontario, London, Ontario, Canada, ${ }^{2}$ Imaging Program, Lawson Health Research Institute, London, Ontario, Canada, ${ }^{3}$ Division of Cardiology, London Health Sciences Centre, London, Ontario, Canada, ${ }^{4}$ Department of Nuclear Medicine, St. Joseph's Hospital, London, Ontario, Canada

Previously, we have developed a clinically translatable nuclear imaging method to track the viability of myocardially transplanted cells in a canine myocardial infarction (MI) model (J Nucl Med. 2009;50:927-935). Inflammation post-MI is necessary for infarct healing but may negatively influence cell viability after transplantation. Cellular subsets of the inflammatory process actively metabolize FDG; therefore in a canine model of MI we propose to use FDG-PET as a method to monitor the inflammatory flux and use this information to understand the best time to transplant cells for therapy using our developed method. Methods: Suppression of FDG uptake in normal myocardium was tested in canines under two conditions $(\mathrm{n}=5)$ : special (high fat/low carbohydrate) vs. normal diet. Within each condition, canines were either fasted overnight prior to PET or supplemented with a fatty liquid on the day of PET (18-F FDG: $309.6 \pm 5.8 \mathrm{MBq}$ ). In permanently infarcted canines $(n=2)$ FDG-PET $(288.2 \pm 6.1 \mathrm{MBq})$ was assessed days 4 , 7 , and 14 following fatty supplementation post-MI. On day 14 , ex vivo delayed enhancement MRI was acquired along with ex vivo FDG-PET and coregistered offline. Results: In normal myocardium, FDG uptake following suppression by fatty supplementation was lowered in 6 of 10 comparisons,
4 of which were significant $(\mathrm{p}<0.05)$, and the special diet did not significantly reduce the FDG uptake relative to normal diet. In infarcted tissue, PET images indicated enhanced uptake over $2 \mathrm{wks}$ in the anterior LV wall peaking at 4 and 7 days. Coregistered DE-MRI with FDG-PET indicated regions of enhanced uptake in the anterior LV wall coinciding with hyperenhanced regions on MR images. Histological staining on day 14 verified infarct-related inflammation in fibrotic myocardium relative to noninfarcted tissue. Conclusion: Despite the variability in adequately suppressing myocardial FDG uptake, FDG-PET shows promise as a method to characterize the inflammatory flux in the anterior LV wall potentially providing insights into the interaction between inflammation and transplanted cell viability.

\section{3}

Effect of aging and smoking on FDG-uptake in atherosclerotic plaques assessed by multiple-time-point PET/CT imaging. B.A. Blomberg ${ }^{1}$, M.H. Gharavi ${ }^{1}$, B. Saboury ${ }^{1}$, T.J. Werner ${ }^{1}$, D. Torigian ${ }^{1}$, G. Cheng ${ }^{2}$, E. Lim ${ }^{2}$, S.R. Akers ${ }^{2}$, A. Alavi ${ }^{1}$; ${ }^{1}$ Department of Radiology, University of Pennsylvania, Philadelphia, Pennsylvania, ${ }^{2}$ Department of Radiology, Philadelphia VA Medical Center, Philadelphia, Pennsylvania

Our objective was to prospectively determine the relation between aging, smoking, and FDG-uptake in atherosclerotic plaque in the aorta by multipletime-point (MTP) imaging. Methods: Ten out of 31 patients with lung cancer were included in this analysis. Each patient underwent MTP PET/CT imaging at one, two, and three hours after FDG administration. FDG-uptake in atherosclerotic plaques, quantified as weighted average SUVmean (aSUVmean) [summation (SUVmean $\times$ surface area $\times$ slice thickness) $/$ total vessel volume], was determined in the aorta by placing ROIs around the aortic wall on every slice of the attenuation-corrected PET/CT images. Subtracting the mean venous activity from the arterial activity performed correction for the blood pool activity. A multiple regression model was fitted to correct for possible confounding by cardiovascular risk factors. Results: Preliminary data of 10 patients with an average age of $64 \pm 10$ years (range, 51-80) showed no significant correlation between aging and blood pool-corrected aSUVmean utilizing images acquired at one (beta $=$ $0.006 ; \mathrm{P}=0.2$ ) and two (beta $=0.004 ; \mathrm{P}=0.1$ ) hours after FDG administration. However, a significant correlation was observed on images taken three hours following injection of the compound (beta $=0.008 ; \mathrm{P}=$ 0.045). Furthermore, a significant correlation in FDG-uptake was observed between smokers $(n=7)$ and nonsmokers $(n=3)$ at the first (beta $=0.037$; $\mathrm{P}=0.01$ ), second (beta $=0.059 ; \mathrm{P}=0.005)$, and third hour (beta $=0.042$; $\mathrm{P}=0.009)$ after FDG administration. Conclusion: Aging and smoking result in increased FDG-uptake in atherosclerotic plaque in the aorta. This effect appears to become more significant at three hours after administration of FDG. Delayed uptake of FDG in atherosclerotic plaque and diminishing levels of FDG in the blood likely account for this important observation. These data support the benefits of delayed-time-point PET imaging in assessing atherosclerosis in any setting.

\section{4}

Anti mouse/human VCAM1 nanobodies for SPECT imaging of atherosclerosis. A. Broisat ${ }^{1}$, S. Hernot ${ }^{2}$, J. Toczek ${ }^{1}$, J. De Vos ${ }^{3}$, L.M. Riou ${ }^{1}$, S. Martin ${ }^{1}$, M. Ahmadi ${ }^{1}$, V. Caveliers ${ }^{2}$, S. Muyldermans ${ }^{2}$, T. Lahoutte ${ }^{2}$, D. Fagret $^{1}$, C. Ghezzi ${ }^{1}$, N. Devoogdt ${ }^{2}$; ${ }^{1}$ Radiopharmaceutiques Biocliniques, INSERM, Grenoble, France, ${ }^{2}$ Laboratory of In Vivo Cellular and Molecular Imaging, Vrije Universiteit Brussel, Brussels, Belgium, ${ }^{3}$ Laboratory of Cellular and Molecular Immunology, Brussels, Belgium

The inflammatory process is recognized as a major criterion for defining a vulnerable atherosclerotic plaque. As an inflammatory marker, Vascular Cell Adhesion Molecule-1 (VCAM1) therefore constitutes a relevant target for molecular imaging of such lesions. By combining nanomolar affinities and fast blood clearance, nanobodies represent potential generic radiotracers for cardiovascular molecular imaging. We aimed to generate, radiolabel, and evaluate anti-VCAM1 nanobodies for noninvasive detection of atherosclerotic lesions. Methods: Ten anti-mouse or anti-mouse/human VCAM1 crossreactive nanobodies with nanomolar affinities were generated, radiolabeled with technetium- $99 \mathrm{~m}$, and screened in vitro on mouse and human recombinant VCAM1 proteins and endothelial cells and in vivo in ApoE- 
deficient (ApoE-/-) mice. A nontargeting control nanobody was used in all experiments to demonstrate specificity. Results: The lead compound was identified as nanobody cAbVCAM1-5. As demonstrated by surface plasmon resonance (SPR), as well as by flow cytometry, cAbVCAM1-5 was found cross-reactive for mouse/human VCAM1 with $\mathrm{Kd}$ of $2.0 \pm 0.0 \mathrm{nM}$ and $6.5 \pm 0.7 \mathrm{nM}$, respectively. In addition, it exhibited high lesion-tocontrol $(4.95 \pm 0.85)$, lesion-to-heart $(8.30 \pm 1.11)$, and lesion-to-blood ratios $(4.32 \pm 0.48)(\mathrm{P}<0.05$ vs control $\mathrm{C} 57 \mathrm{Bl} / 6 \mathrm{~J}$ mice for all 3 ratios $)$. Atherosclerotic lesions located within the aortic arch of ApoE-/- mice were successfully identified by SPECT/CT imaging, and ${ }^{99 \mathrm{~m}} \mathrm{Tc}-\mathrm{cAbVCAM} 1-5$ binding specificity was further demonstrated by in vivo competition experiments. Autoradiography and immunohistochemistry further confirmed cAbVCAM1-5 uptake in VCAM1-positive lesions. Conclusion: The ${ }^{99 \mathrm{~m}}$ Tc-labeled, anti-VCAM1 nanobody cAbVCAM1-5 allowed noninvasive detection of VCAM1 expression and displayed mouse and human cross reactivity. Therefore, this study demonstrates the potential of nanobodies as a new class of radiotracers for cardiovascular applications. The nanobody technology might evolve into an important research tool for targeted imaging of atherosclerotic lesions and has the potential for fast clinical translation.

\section{5}

Improved image reconstruction for dedicated cardiac SPECT with truncated projections. C. Chan ${ }^{1}$, J. Dey ${ }^{2}$, A. Sinusas ${ }^{1}$, C. Liu ${ }^{1}$; ${ }^{1}$ Yale University, New Haven, CT, ${ }^{2}$ University of Massachusetts Medical School, Worcester, MA

The small field-of-view (FOV) of dedicated cardiac SPECT can cause projections truncated by radiotracer in organs outside the FOV. This may lead to artifacts and overestimation in quantifications. In this study, we investigated the impact of different image matrix sizes on reconstruction and propose using body contour obtained from CT to improve reconstruction on a multiple-pinhole small-FOV SPECT/CT system. Methods: We simulated the system with 27 pinholes arranged on a cylindrical surface that covers 180 degree of the body. The FOV of the system was $21 \mathrm{~cm}$ in diameter. We compared MLEM reconstruction with an image matrix of $60 \times$ $60 \times 128$ that covers just the FOV (IM60), $80 \times 80 \times 128$ that covers the majority portion of the body (IM80), a large image matrix of $128 \times 128 \times$ 128 with body contour (LIMBC) obtained from CT images that constrains the reconstruction, and the same matrix without constraints (LIM). Noiseless data and 20 noise realizations with $\sim 230 \mathrm{~K}$ counts per projection of a NCAT phantom with defects were simulated. Quantitative accuracy was evaluated in terms of bias versus noise, and contrast recovery. Results: The reconstructions with IM80, LIMBC, and LIM reduced the artifacts in the myocardium and blood pool caused by projection truncations. The loglikelihood analysis shows that all reconstructions converged to the true likelihood except IM60, and LIMBC has the fastest convergence rate. For quantitative evaluations, IM60 yielded accurate results in myocardium, but $\sim 20 \%$ and $\sim 115 \%$ overestimation in defect and blood pool, respectively, which led to reduced contrast recovery. LIMBC yielded the most accurate quantification, where LIM yielded up to $10 \%$ underestimation of regional counts in the myocardium. Conclusion: Our results indicate that application of an image matrix that matches with the body contour provides the most accurate reconstruction for small-FOV multiple-pinhole SPECT, while a smaller image matrix can cause artifacts and overestimation, and an arbitrary large image matrix can lead to an underestimation of counts.

\section{6}

Simultaneous respiratory and cardiac motion corrections for a dedicated cardiac SPECT system. C. Chan ${ }^{1}$, L. Volokh ${ }^{2}$, M. Harris ${ }^{3}$, M. $\mathrm{Le}^{3}$, J. Biondi ${ }^{3}$, A. Sinusas ${ }^{1}$, C. Liu ${ }^{1} ;{ }^{1}$ Yale University, New Haven, CT, ${ }^{2} \mathrm{GE}$ Healthcare, Haifa, Israel, ${ }^{3}$ Ivy Biomedical Systems, Branford, CT

Respiratory and cardiac motions can degrade the myocardial perfusion SPECT image quality and quantitative accuracy. In this study, we developed methods to simultaneously correct respiratory and cardiac motions for a stationary dedicated cardiac SPECT to improve the image quality and quantifications. Methods: The respiratory motion was monitored by a compressive sensor pillow attached to the phantom or patient's lower chest and connected to a dual respiratory-cardiac gating box (Ivy Biomedical Systems), which sends cardiac triggers to GE Discovery 530c/570c systems only during end-expiration phases. The list-mode data was rebinned retrospectively into end-expiration frames only for respiratory motion correction or 8 cardiac gates only during end-expiration phases to correct for both respiratory and cardiac motions. A nonrigid registration method was also used to register all dual-gated images to a reference gate to reduce noise while retaining motion reduction. The respiratory trace acquired on the dual gating box was first validated against an Anzai system. Image quality improvement was evaluated using a torso phantom driven by a motion platform with real patient traces and patient data in terms of myocardium-to-blood pool contrast and visual assessment. Results: The traces acquired using the sensor pillow matched well with the Anzai system. In phantom study, end-expiration gating reduced motion blurring and increased myocardium-to-blood pool contrast from 2.3 in the ungated image to 6.2 , which is close to 6.6 in the motion-free image. Additional cardiac gating in the patient study further reduced the blurring effect and revealed fine structures at the expense of increased noise. The nonrigid registration of images from 8 cardiac gates improved tradeoff between motion correction and noise increase. Conclusion: The proposed methods can effectively reduce both respiratory and cardiac motions and may lead to more accurate defect detection and quantifications.

\section{7}

What is the interobserver variability of ${ }^{18}$ F-FDG PET/CT carotid imaging? A sub-study of the Canadian Atherosclerosis Imaging Network (CAIN). M.S. Cocker ${ }^{1}$, B. McArdle ${ }^{1}$, R. deKemp ${ }^{1}$, C. Lum², G.

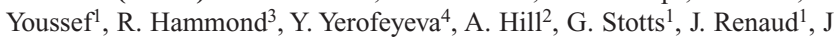
Brennan $^{1}$, J. DaSilva ${ }^{1}$, J.C. Tardif ${ }^{5}$, J.D. Spence ${ }^{6}$, R. Beanlands ${ }^{1} ;{ }^{1}$ University of Ottawa Heart Institute, Ottawa, Ontario, Canada, ${ }^{2}$ The Ottawa Hospital, Ottawa, Ontario, Canada, ${ }^{3}$ Schulich School of Medicine and Dentistry, London Health Sciences Centre and University of Western Ontario, London, Ontario, Canada, ${ }^{4}$ Sunnybrook Hospital, Toronto, Ontario, Canada, ${ }^{5}$ Montreal Heart Institute, Montreal, Quebec, Canada, ${ }^{6}$ University of Western Ontario, London, Ontario, Canada

Positron emission tomography (PET) using $\left[{ }^{18} \mathrm{~F}\right]$-fluorodeoxyglucose (FDG) may identify inflamed plaque in carotid vasculature. It is important to determine the maximal FDG uptake: i) clinically, ii) for comparison to histology, and iii) because FDG uptake is being utilized as an endpoint in emerging clinical trials. Therefore, in this study, we sought to determine the reproducibility of quantifying FDG uptake in internal carotid arteries. Methods: Twenty-two lesions were identified in eleven prospectively recruited patients (10 male, $68 \pm 11$ years) scheduled for carotid endarterectomy. Patients underwent hybrid FDG PET and computed tomography angiography (CTA). Two independent observers evaluated PET/CTA images. The bifurcation of the common carotid artery was located, and the region of maximal FDG uptake at or above the bifurcation or along the internal carotid artery was identified. Results: Strong interobserver agreement for the quantification of maximum FDG uptake was noted. The ICC was 0.911 , and the linear correlation coefficient was $\mathrm{r}=$ $0.830, \mathrm{p}<0.001$. Conclusion: PET/CTA imaging enables reproducible quantification of maximum FDG uptake. This approach is promising diagnostically and as a means to monitor therapy.

\section{8}

Detection of atherosclerotic lesions using a novel small peptide based imaging probe targeted at the lectin-like oxidized LDL receptor. $\mathrm{J}$. Dimastromatteo $^{1}$, K.A. Kelly ${ }^{1}$, S. Thomas ${ }^{1}$, A. Klibanov ${ }^{1}$, D.K. Glover ${ }^{1}$; ${ }^{1}$ University of Virginia, Charlottesville, VA

The lectin-like oxidized LDL receptor (LOX-1) is known to play an important role in the initiation, progression, and destabilization of atherosclerotic plaques. We previously demonstrated the feasibility of detecting lesions associated with markers of plaque vulnerability using a LOX-1 targeted liposomal mAb construct. The objective of this study was to develop and validate a directly labeled small peptide imaging probe targeted at the LOX-1 receptor in a murine model of atherosclerosis. Methods: A small peptide targeting both murine and human LOX-1 with high affinity and selectivity was identified by phage display screening. A dimeric form of the LOX-1 binding peptide was synthesized and radiolabeled with Tc99m using an Isolink ${ }^{\circledR}$ kit (LBP). LBP was injected i.v. in ApoE-/- $(60$ weeks, $n=4)$ and in WT $(8$ weeks, $n=4)$ mice and blood 
samples collected over 2 hrs. Postmortem excision of aortas and other organs was performed. Aortas were cleaned of fat and adventitia, opened longitudinally and exposed overnight on a phosphor imaging plate, followed by Sudan-IV staining. Biodistribution of LBP was measured by gamma well counting. Results: Radiolabeling efficiency was $95 \%$. In vitro, LBP demonstrated specific binding to rhLOX-1. LBP blood pool clearance was rapid, with $<5 \%$ initial activity at $2 \mathrm{hrs}\left(\mathrm{t}^{1} / 2 \sim 8 \mathrm{~min}\right)$. Tracer excretion was predominantly renal. There was 3 -fold higher uptake of LBP in the aortas of ApoE $-/-$ vs WT mice $(0.17 \pm 0.06$ vs $0.06 \pm 0.02 \% \mathrm{ID} / \mathrm{g}$, respectively, $\mathrm{p}<$ $0.05)$. Focal LBP uptake was observed on ex vivo images in Sudan-IV positive regions on the aortas of ApoE- - - mice, whereas there was minimal LBP uptake in the aortas of WT mice without significant plaque. By quantitative analysis of images, there was 2-fold higher uptake of LBP in the ApoE-/- mice vs WT $(1.88 \pm 0.07$ vs $1.03 \pm 0.05$, respectively, $\mathrm{p}<0.05)$. Conclusion: LBP targeted atherosclerotic plaques in vivo, showing excellent correlation with histology and favorable blood clearance and biodistribution properties. Further studies are needed to determine the potential role of LBP for identifying vulnerable patients.

\section{9}

Development of LOX-1 nanobodies for imaging of atherosclerosis. J. De Vos $^{1,2}$, A. Broisat ${ }^{3}$, J. Toczek ${ }^{3}$, C. Ghezzi ${ }^{3}$, S. Muyldermans ${ }^{1}$, T. Lahoutte ${ }^{2}$, N. Devoogdt ${ }^{2} ;{ }^{1}$ Laboratory of Cellular and Molecular Immunology, Vrije Universiteit Brussel, Brussels, Belgium, ${ }^{2}$ Laboratory of In Vivo Cellular and Molecular Imaging, Vrije Universiteit Brussel, Brussels, Belgium, ${ }^{3}$ Radiopharmaceutiques Biocliniques, INSERM, Grenoble, France

Nanobodies are the smallest antigen-binding fragments derived from unique camelid antibodies lacking light chains and are particularly suited as probes for in vivo imaging. Lectin-like Oxidized LDL Receptor 1 (LOX1) is a receptor for oxidized LDL and is a marker of the vulnerable atherosclerotic plaque. The objective of this study is to develop nanobodies targeting LOX-1 for the detection of atherosclerotic lesions in preclinical animal models of atherosclerosis. Methods: A dromedary was immunized with both human (hLOX-1) and mouse LOX-1 (mLOX-1) recombinant proteins. An immune nanobody library was generated, phage-displayed, and biopannings were performed on recombinant mLOX-1 and hLOX-1. Screening of nanobodies was performed via ELISA on both recombinant proteins. The affinities of the nanobodies for mouse and/or human recombinant LOX-1 and the targeted epitope were evaluated using Surface Plasmon Resonance. The nanobodies targeting $\mathrm{mLOX}-1$ were labeled with ${ }^{99 \mathrm{~m}} \mathrm{Tc}$ and injected in $\mathrm{APOE}^{-/-}$mice and in control C57B16 mice to evaluate their plaque targeting using SPECT/CT imaging. After euthanasia, organs and tissues were counted in a gamma counter to determine the biodistribution ex vivo. Results: Nanobodies binding different epitopes on either mouse or human recombinant LOX-1 with nanomolar affinity were generated. Cross-reactive nanobodies were identified; however none of them had a nanomolar affinity for both mouse and human LOX-1. This could be explained by the relatively low homology between both LOX-1 proteins. After ${ }^{99 \mathrm{~m}} \mathrm{Tc}$ labeling the nanobodies still bound $\mathrm{mLOX}-1$. The anti-mLOX-1 nanobody with the best affinity $(0.4 \mathrm{nM})$ showed only moderate uptake in atherosclerotic lesions in the aortic arch of $\mathrm{ApoE}^{-/}$ mice using SPECT/CT imaging. The ex vivo uptake $(0.91 \pm 0.12 \%$ injected activity/g) was higher than the uptake in aortas of C57B16 control mice $(0.51 \pm 0.09 \%$ injected activity/g). Conclusion: Nanobodies binding recombinant mouse and human LOX-1 with nanomolar affinity were generated. One nanobody targeting mLOX-1 showed a relatively low in vivo uptake in aortic atherosclerotic lesions. We conclude that nanobodies selected for strong binding to recombinant LOX-1 protein are not optimal for in vivo detection of LOX-1 expression, possibly due to structural differences between LOX-1 recombinant protein and LOX-1 expressed in atherosclerotic lesions. In future experiments nanobodies will be selected on cells that express mouse and human LOX-1.

\section{$10^{*}$}

3D acquisitions improve sensitivity and coverage in cardiac F19 imaging. S.W. Fielden ${ }^{1}$, A.L. Klibanov ${ }^{1}$, F.H. Epstein ${ }^{1}$, Y. Xu ${ }^{1}$, B.A. French ${ }^{1}$, C.H. Meyer ${ }^{1}$, ${ }^{1}$ University of Virginia, Charlottesville, VA

*Young Investigator Travel Award winner.
Traditional MRI contrast agents generate local signal level changes in proton images. However, for small agent concentrations the percent change in signal can be very small, necessitating pre- and postcontrast images, which increases the overall study time as well increasing the chance of artifacts due to between-scan motion. For these reasons, secondcolor imaging with MRI is under increasing development. Fluorine, in particular, is an attractive second nucleus for MRI, but localization of fluorine can be difficult due to the inherent low sensitivity of MRI. Threedimensional imaging has known sensitivity advantages over 2D imaging in MRI. Furthermore, 3D imaging can provide extended coverage over which signal may be detected. The objective of this study was to demonstrate that low-SNR cardiac fluorine imaging benefits from a gated 3D acquisition. Methods: Myocardial infarction was surgically induced in $\mathrm{C} 57 \mathrm{Bl} / 6$ mice by a one-hour occlusion followed by reperfusion. A 300-microliter volume of either in-house-manufactured or commercially obtained (VS-1000H) nanoparticles was injected intravenously 24-hours postsurgery. Imaging was performed 4 and 8 days postsurgery to allow time for macrophage phagocytosis of nanoparticles and recruitment into damaged myocardium. The study was performed on a Bruker 7-T smallanimal system with a dual-tuned ${ }^{19} \mathrm{~F} /{ }^{1} \mathrm{H}$ coil. In addition to $2 \mathrm{D}$ fluorine acquisitions that have been demonstrated for this application (Flögel et al. Circulation. 2008;118:140-148), a gated 3D turbo-spin-echo sequence was applied with coverage extending over the entire coil volume. Late gadolinium-enhanced (LGE) images were obtained in order to localize areas of myocardial scarring. Results: Volumetric fluorine images were obtained in 11:44 minutes and are comparable to $2 \mathrm{D}$ images obtained in 19:12 minutes. Fluorine signal was observed in areas of myocardial infarct using both nanoparticle formulations. Additionally, nanoparticle accumulation was observed in the spine and long bones of the mice using the 3D acquisition. Conclusion: The sensitivity advantage of 3D encoding allowed a greater volume to be imaged in less time than multislice 2D, with similar image quality. Gating is necessary to mitigate artifacts from cardiac and respiratory motion. The use of whole-mouse imaging allowed observation of contrast accumulation in the spine and long bones; this result was unanticipated and might have gone unobserved without the use of whole-mouse imaging.

\section{$11^{*}$}

Noninvasive tracking of human mesenchymal stem cells in vivo using imaging-visible microcapsules and triple-fusion reporter. $\mathrm{Y}$. $\mathrm{Fu}^{1}, \mathrm{R}$. Mease $^{1}$, Y. Chen ${ }^{1}$, M. Solaiyappan ${ }^{1}$, J. Cook ${ }^{1}$, D.L. Kraitchman ${ }^{1} ;{ }^{1}$ Johns Hopkins University, Baltimore, MD

Therapeutic angiogenesis using stem cells has shown promise for treating ischemic arterial disease in both experimental and clinical settings. However, its efficacy is limited by poor cell retention, low cell survival rate, and the lack of the ability to track cell fate noninvasively in vivo. In this study, we investigate the potential of coupling imagingvisible cell microencapsulation techniques with triple-fusion (TF) reporter gene labeling for human mesenchymal stem cells (hMSCs) tracking with PET-CT. Methods: Bone marrow-derived hMSCs were stably transfected with a lentiviral vector encoding firefly luciferase (fluc, bioluminescence imaging [BLI] reporter), red fluorescence protein $(r f p)$, and truncated thymidine kinase ( $h s v_{t k}$, PET reporter). BLI was acquired before and after cell encapsulation to ensure proper gene transduction (Xenogen IVIS 2000). Alginate microencapsulation of TF-hMSCs was performed using a modification to incorporate perfluorooctylbromide (PFOB) to enable $\mathrm{x}$-ray visibility. $\left[{ }^{18} \mathrm{~F}\right]$-labeled 9-[4-fluoro-3-(hydroxymethy) butyl] guanine derivative ( $\left[{ }^{18} \mathrm{~F}\right]$-FHBG) was synthesized using a previously described method. For in vivo studies, NZW rabbits received 4-6 injections of PFOB encapsulated TF-hMSCs in the medial thigh, and a c-arm CT image was obtained prior to $\left[{ }^{18} \mathrm{~F}\right]$-FHBG administration ( 1.7 mCi). Dynamic PET imaging (HRRT) was acquired $60 \mathrm{~min}$ after radiotracer administration for a period of $90 \mathrm{~min}$. The "hot spot" PET images were coregistered with c-arm CT images to identify the location of transplanted cells using a 3D visualization software (Dextroscope). One week after delivery, BLI was performed ex vivo on microcapsules dissected from the rabbit thigh to assess cell viability. Results: In vitro hMSC viability and transgene expression remained after encapsulation. In vivo, all $\mathrm{PFOB}$ cap injections were detected on c-arm CT images. Using PET, transplanted PFOB 
microcapsules in rabbit medial thigh were identified as "hot spots" without background and showed high concordance to the radiopacities on c-arm CT. BLI demonstrated viable xenogeneic TF-hMSCs in PFOB microcapsules 1 week postdelivery. Conclusion: We present the first demonstration of xenogeneic MSC delivery in nonimmunosuppressed animals using novel perfluorinated microcapsule and reporter gene labeling. PFOB microencapsulation of TF-hMSCs maintains cell viability in vivo and provides a means to assess cell viability and enable cell tracking using clinical PET-CT.

\section{2}

Human hepatocyte growth factor infected bone marrow mesenchymal stem cells for myocardial infarction: assessment with 7.0T MR imaging. Y.K. Guo ${ }^{1}$, Z.L. Li ${ }^{2}$, H.Z. Chen ${ }^{1}$, R. Xia ${ }^{2}$, J.L. Hou ${ }^{3}$, N. Gang ${ }^{4}$, F.B. Gao ${ }^{1}$ Department of Medical Imaging, West China Second University Hospital, Sichuan University, Chengdu City, Sichuan Province, China, ${ }^{2}$ Department of Radiology, West China Hospital, Sichuan University, Chengdu City, Sichuan Province, China, ${ }^{3}$ Department of Thoracic and Cardiovascular Surgery, West China Hospital, Sichuan University, Chengdu City, Sichuan Province, China, ${ }^{4}$ Chengdu City, Sichuan Province, China

Our objective was to prospectively determine the effects of intramyocardial injection of lentiviral vector carrying the human hepatocyte growth factor (hHGF) infecting the bone marrow mesenchymal stem cells (BMSCs) on intervening myocardial infarction (MI), and to monitor in vivo with cardiovascular magnetic resonance (MR) imaging. Methods: The institutional animal care and use committee approved this study. BMSCs were labeled by superparamagnetic iron oxide (SPIO). MI was successfully induced in 40 Sprague-Dawley rats by ligation of the left coronary artery. Then 40 rats were randomly assigned to 5 groups: 3 treated groups included hHGF infecting BMSCs group, only hHGF group, and only BMSCs group; the other groups included injection of pcDNA group (control group) and sham group (without coronary ligation). MR (7.0 T, Bruker BioSpec 70/30, Germany) microimaging system with rat heart coils was used to trace the BMSCs and measure the perfusion, infarct size, wall motion, and LV ejection fraction. TTC stains, Masson trichrome stains, and immunohistochemistry were used to assess the infarct area and regional vascular density. One-way ANOVA test was used to determine significant differences between animal groups. Bonferroni correction was used to adjust $\mathrm{p}$ values for multiple comparisons. Results: Before therapy, MR imaging measurements of LV function and perfusion showed no difference among all the groups $(\mathrm{p}>0.05)$ but the sham group in acute MI. After therapy in subacute infarction, cardiac MR imaging could monitor the BMSCs labeled by SPIO in vivo. In chronic infarction, the infarct areas in the treated groups reduced at perfusion and delayed-enhancement imaging $(\mathrm{p}<$ 0.05 ), and ejection fractions increased when compared with the control group. Bonferroni comparison, moreover, revealed that the best therapeutic effects of hHGF infecting BMSCs group was obtained among the treated groups. In contrast, the infarct area and LV function deteriorated further in the control group. Histopathologic findings and microvessel counts in the treated groups were significantly better than in the control $(p=0.02)$. Conclusion: Intramyocardial injection of hHGF infecting BMSCs significantly improved cardiac functions, reduced infarct area, and stimulated angiogenesis. MR imaging may be helpful to dynamically monitor and assess the therapeutic effects of myocardial infarction in vivo.

\section{3}

Targeting VEGF expression to identify accelerated atherosclerosis. M. Kollaros $^{1}$, G. Zhang ${ }^{1}$, M.V. Backer ${ }^{2}$, J.M. Backer ${ }^{2}$, L.L. Johnson ${ }^{1}$; ${ }^{1}$ Columbia University, New York, NY, ${ }^{2}$ SibTech, Inc., Brookfield, CT

The biology of the vulnerable plaque includes increased inflammation and rapid growth of vasa vasorum, processes that are characterized by vascular endothelial growth factor (VEGF) expression in response to hypoxia and inflammation and are accelerated in diabetes. We hypothesized that a novel radiolabeled probe ${ }^{99 \mathrm{~m}} \mathrm{Tc}$ scVEGF-PEG-DOTA) targeting VEGFR1 and VEGFR2 can identify accelerated atherosclerosis in diabetes.
Methods: At 6 wks of age, 5 apoE null mice were made diabetic with streptozotocin (DM) and 4 were not treated (NDM). At 25 wks of age, all mice were injected with $500 \mu \mathrm{Ci}{ }^{99 \mathrm{~m}} \mathrm{Tc}$ scVEGF-PEG-DOTA and 2-3 hr later (blood pool clearance) injected with eXIA CT contrast agent and underwent SPECT/CT imaging (Bioscan). At completion of imaging mice were euthanized. The chest was opened, and the aortic arch and great vessels were dissected out, photographed in-situ, removed, paraffin embedded, and sectioned and stained for VEGFR1 VEGFR2, macrophages (mac-3), and endothelial cells (factor VIII). Organs were harvested and counted in the well counter to determine biodistribution. ROIs were drawn around tracer localized to aortic root and arch from CT scan, and tracer uptake was quantified as total and mean activity/voxel in $\mu \mathrm{Ci}$ (InVivoScope software). ROIs were also placed in the pulmonary vein for mean activity/ voxel background. Results: All 9 mice showed focal uptake of tracer localized by the CT contrast to the aortic root, arch, and neck vessels. Results were the following: Target to bknd was high for both DM and $\operatorname{NDM}(3.27 \pm 0.89$ and $4.46 \pm 1.49)(\mathrm{P}=0.18)$ while the total uptake (in $\left.\mu \mathrm{Ci} \times 10^{-2}\right)$ was significantly higher for DM than NDM $(16.6 \pm 6.3$ vs. $5.9 \pm$ 2.6) $(\mathrm{P}=0.02)$. Staining for VEGFR and macs as + cells/vascular section was higher in DM than in NDM (1844 vs. 697 and 2222 vs. 996, respectively). These results show good T/B uptake, with more extensive uptake in DM compared to NDM. Staining for ECs showed staining confined to endovascular lining. Immunofluorescence studies show colocalization of VEGR1 predominantly with macrophages. Conclusion: ${ }^{99 \mathrm{~m}} \mathrm{Tc}$ scVEGF-PEG-DOTA shows promise as a noninvasive approach to identify vulnerable plaque. In this model, VEGF expression was predominantly in macrophages and showed increased expression in diabetes.

\section{4}

Comparison of two methods of stem cell delivery into the myocardium. L. Lezaic ${ }^{1}$, A. Socan ${ }^{1}$, B. Vrtovec ${ }^{2}$, G. Poglajen ${ }^{2}$, M. Sever ${ }^{3}$, D. Domanovic $^{4}$, J. Fettich ${ }^{1} ;{ }^{1}$ Department for Nuclear Medicine, University Medical Centre Ljubljana, Ljubljana, Slovenia, ${ }^{2}$ Cardiology Department, Advanced Heart Failure Centre, University Medical Centre Ljubljana, Ljubljana, Slovenia, ${ }^{3}$ Hematology Department, University Medical Centre Ljubljana, Ljubljana, Slovenia, ${ }^{4}$ Blood Transfusion Centre, Ljubljana, Slovenia

Stem cell transplantation is an emerging method in treatment of advanced heart failure. The amount and retention of stem cells after myocardial delivery may likely influence treatment efficiency. Our objective was to compare myocardial retention of transplanted stem cells after myocardial delivery by two different methods. Methods: Patients with advanced heart failure (NYHA III/IV, heart transplant candidates) on optimal medical treatment and stable symptoms during the last 6 months were included. Bone marrow was stimulated by G-CSF, stem cells were collected by peripheral blood apheresis, and the hematopoietic stem cell portion (HSC, CD34+) was immunomagnetically selected. Predefined volume $(20 \%)$ of injectate $(100 \mathrm{ml}$ for intracoronary [IC] route, $10 \mathrm{ml}$ for intramuscular [IM] route) was used for HSC labeling with ${ }^{99 \mathrm{~m}} \mathrm{Tc}-\mathrm{HMPAO}$ and resuspended with the remaining injectate after labeling. Labeled stem cells were tested for labeling efficiency, stability, and viability. In 34 patients, HSC were injected IC, and target vessel was selected by myocardial perfusion imaging (MPI, gated SPECT) prior to transplantation as one supplying viable yet dysfunctional myocardium. In 19 patients, HSC were injected intramyocardially (IM) into viable yet dysfunctional areas as assessed by MPI, ${ }^{18} \mathrm{FDG}-\mathrm{PET} / \mathrm{CT}$, and confirmed directly prior to injection by NOGA XP Cardiac navigation system. Planar imaging of the chest and upper abdomen was performed $1 \mathrm{~h}$ after HSC transplantation for quantification using conjugate view method after background correction $(128 \times 128$ matrix, $10 \mathrm{~min}$ per projection, $\mathrm{AP} / \mathrm{PA}, \mathrm{LAO} / \mathrm{RPO}$ projections $)$, followed by tomographic imaging of the thorax. Planar imaging was repeated next morning (approximately $18 \mathrm{~h}$ p.i.). Results: In IC group, average $\mathrm{HSC}$ retention in the myocardium was $0.7 \% 1 \mathrm{~h} \mathrm{p}$.i. and $0.5 \% 18$ $\mathrm{h}$ p.i. ( $<00.001$, paired t-test). In IM group, average HSC retention at $1 \mathrm{~h}$ p.i. was $3.9 \%$ and at $18 \mathrm{~h}$ p.i. was $2.9 \%$ (paired t-test, $\mathrm{p}<0,001$ ). At both time points, difference/ratio of IM vs. IC delivery route retention (5.6 at $1 \mathrm{~h} \mathrm{p}$.i. and $5.818 \mathrm{~h}$ p.i.) was statistically significant (unpaired t-test, $\mathrm{p}<$ 0.0001). Conclusion: IM method of HSC delivery into the myocardium appears to be more effective than the IC route. 
Dual isotope hybrid SPECT/CT imaging permits serial quantitative assessment of matrix metalloproteinase activity following myocardial infarction. B.A. Lin ${ }^{1}$, M.R. Stacy ${ }^{1}$, C. Chan ${ }^{1}$, M.W. Maxfield ${ }^{1}$, B.P. Jozwik $^{1}$, D.P. Dione ${ }^{1}$, X. Wang ${ }^{1}$, H. Lakhani ${ }^{1}$, L. Volokh ${ }^{2}$, Y.-H. Liu ${ }^{1}$, J.S. Duncan ${ }^{1}$, C. Liu ${ }^{1}$, A.J. Sinusas ${ }^{1}$; ${ }^{1}$ Yale University, New Haven, CT, ${ }^{2}$ GE Healthcare, Haifa, Israel

Matrix metalloproteinase (MMP) activation modulates left ventricular (LV) remodeling following myocardial infarction (MI). We previously demonstrated time-dependent changes in myocardial MMP activity in the MI region by postmortem gamma well counting of a ${ }^{99 \mathrm{~m}} \mathrm{Tc}$-labeled MMP inhibitor ( $\left.{ }^{99 \mathrm{~m}} \mathrm{Tc}-\mathrm{RP} 805\right)$ injected in animals sacrificed at different time points after surgically induced MI. The objective of this study was to evaluate serial in vivo changes in MMP activity within the MI region in relation to changes in LV end-diastolic (ED) volume through quantitative assessment of myocardial ${ }^{99 \mathrm{~m}} \mathrm{Tc}-\mathrm{RP} 805$ activity and contrast CT using hybrid SPECT/CT and to relate these indices to postmortem MI size. We hypothesized that the degree of early MMP activation within the MI would predict late post-MI remodeling. Methods: In vivo hybrid dual isotope SPECT and 64-slice CT imaging (Discovery NM-CT 570c, GE Healthcare) was performed in $\operatorname{dogs}(n=5)$ before, and after 3 days and 4 weeks following, a 6 hour angioplasty occlusion of the LAD. SPECT imaging was performed 2 hours following the intravenous injection of ${ }^{99 \mathrm{~m} T c-R P 805}$ and $15 \mathrm{~min}$ after injection of thallium-201. X-ray CT angiography with iodinated contrast was performed to define coronary anatomy and LV ED volumes. Relative retention of ${ }^{99 \mathrm{~m}} \mathrm{Tc}-\mathrm{RP} 805$ within the LV infarct region was quantitatively assessed using Xeleris software (GE Healthcare). The CT images facilitated the serial assessment of the same myocardial regions over time. The \%MI was calculated from postmortem analyses. Results: MI size was variable between dogs (range: $1 \%$ to $19 \% \mathrm{LV}$ ). In vivo SPECT/CT imaging established the temporal changes in ${ }^{99 \mathrm{~m}} \mathrm{Tc}-\mathrm{RP} 805$ relative retention (MI/normal ratio) within the MI (baseline: $0.95 \pm 0.14 ; 3$ day: $2.4 \pm 1.16^{*} ; 4$ week: $1.09 \pm 0.30,{ }^{*} p<$ 0.05 vs. baseline $)$. ED volumes increased from baseline $(60.9 \pm 5.9 \mathrm{ml})$ to 4 weeks post-MI $(67.3 \pm 9.4 \mathrm{ml})$. Relative ${ }^{99 \mathrm{~m}} \mathrm{Tc}-\mathrm{RP} 805$ retention in MI at 3 days correlated with final MI size $\left(\mathrm{R}^{2}=0.72\right)$ and 4 week ED volume $\left(\mathrm{R}^{2}=0.96\right)$. In comparison, final MI size did not correlate as strongly with 4 week ED volume $\left(\mathrm{R}^{2}=0.78\right)$ as early MMP activation did. Conclusion: ${ }^{99 \mathrm{~m}} \mathrm{Tc}-\mathrm{RP} 805 \mathrm{SPECT} / \mathrm{CT}$ imaging provides a useful noninvasive method for serial quantification of regional MMP activation within MI. Early MMP activation within the MI predicted subsequent post-MI remodeling.

\section{$16^{\star}$}

Therapeutic efficacy of different liposomal glucocorticoids as a treatment for atherosclerosis assessed by multimodal imaging. M.E. Lobatto $^{1}$, M.J. Otten ${ }^{1}$, C. Calcagno ${ }^{1}$, S. Ramachandran ${ }^{1}$, D.F. van Wijk ${ }^{2}$, G. Storm $^{3}$, E.S. Stroes ${ }^{2}$, Z.A. Fayad ${ }^{1}$, W.J. Mulder ${ }^{1}$, J.M. Metselaar ${ }^{3} ;{ }^{1}$ Mount Sinai School of Medicine, New York, NY, ${ }^{2}$ Academic Medical Center, Amsterdam, The Netherlands, ${ }^{3}$ Utrecht University, Utrecht, The Netherlands

We previously established that liposomal prednisolone phosphate (a glucocorticoid) can target atherosclerotic plaques and exert profound local antiinflammatory activity. In this study we aimed to evaluate the therapeutic efficacy of different formulations of liposomal glucocorticoids (budesonide $1 \mathrm{mg} / \mathrm{kg}$, dexamethasone $1 \mathrm{mg} / \mathrm{kg}$, and prednisolone phosphate $10 \mathrm{mg} / \mathrm{kg}$ ) by multimodal noninvasive imaging with the purpose of finding a lead formulation for future human clinical trials. In addition, we assessed pharmacokinetics, biodistribution, and toxicity of the drugs. Methods: 36 New Zealand white rabbits received a double balloon injury of the abdominal aorta and a high-fat diet to induce atherosclerotic lesions. After six months animals underwent a pretherapy ${ }^{18} \mathrm{~F}-\mathrm{FDG}$ PET-CT scan and a dynamic contrast-enhanced MRI (DCE-MRI) to assess baseline inflammation and neovascularization, respectively. The rabbits were randomized into four different groups (the different formulations and one saline control group) and were injected with a single intravenous dose of

*Young Investigator Travel Award winner. treatment. All rabbits were imaged 2 days and 7 days after initiating therapy and were subsequently sacrificed for histological assessment. In addition, 18 healthy New Zealand white rabbits were divided into 6 groups $(n=3$ / group) and were injected with the different formulations and their free circulating counterpart. Blood was withdrawn at different time points for pharmacokinetics and clinical chemistry. Rabbits were sacrificed at set end points to assess biodistribution by HPLC. Results: Liposomal prednisolone phosphate caused a significant reduction of PET-CT signal $(16 \%, p=0.03)$ two days postinjection, while a trend towards reduction was seen in animals in other treatment arms. DCE-MRI showed no significant changes in plaque permeability, and MRI-established vessel wall thickness remained unchanged for the different groups. The clinical chemistry in healthy rabbits showed no liver or kidney damage in all groups, while a transient increase in glucose was seen in the first four hours. All free circulating glucocorticoids caused a larger increase of circulating neutrophils and decrease of lymphocytes compared to liposomal glucocorticoids. Monocyte levels remained unchanged. Conclusion: A single intravenous injection of liposomal glucocorticoids can cause a decrease in inflammatory activity monitored by noninvasive multimodal imaging. In addition no significant short-term side effects were witnessed.

\section{7}

A singular value-based approach to real-time targeted molecular imaging in large blood vessels. F.W. Mauldin, Jr. $^{1}$, A.H. Dhanaliwala ${ }^{1}$, A.V. Patil ${ }^{1}$, J.A. Hossack ${ }^{1}$; ${ }^{1}$ University of Virginia, Charlottesville, VA

Ultrasound-based real-time targeted molecular imaging in large blood vessels holds promise for early diagnosis of significant diseases, such as stroke, atherosclerosis, and cancer. At the core of this imaging strategy is the isolation of ligand-receptor bound adherent microbubbles from free microbubbles and tissue structures. Because the frequency spectra of received echo data from these signal components often overlap, current frequency-based techniques (e.g., CPS and harmonic imaging) possess limited performance. We present an alternative approach, termed singular spectrum-based targeted molecular (SiSTM) imaging, which uses statistical information obtained from singular value decomposition to isolate image regions containing adherent microbubbles. Methods: In vitro gelatin flow phantom experiments were performed under physiologically relevant conditions $(2.5 \mathrm{~cm} / \mathrm{s}$ flow velocity and $4-\mathrm{mm}$ diameter). Channels were either targeted to biotinylated $2 \times 10^{6}$ microbubble $/ \mathrm{mL}$ solution with streptavidin or blocked using BSA. Microbubbles were prepared with DiI fluorophore incorporated into the shell for the purpose of optical confirmation of adherent microbubbles. The SiSTM imaging technique was implemented on a programmable research ultrasound scanner using a standard linear array transducer and compared to two alternative techniques - pulse inversion with infinite impulse response filtering (PI + IIR) and harmonic imaging with IIR (Harmonic + IIR). All techniques leveraged acoustic radiation force pulses to enhance binding efficiency and operated on the same received echo data. Results: Experimental SiSTM images produced the expected trend of greater adherent microbubble signal in targeted versus nontargeted microbubble experiments $(\mathrm{P}<0.05, \mathrm{n}=4)$. These results were qualitatively confirmed with en face fluorescent images of DiI signal along the channel wall after SiSTM imaging experiments. In comparison to PI + IIR and Harmonic + IIR techniques, SiSTM provided significantly higher image contrast $(\mathrm{P}<0.001, \mathrm{n}=4)$ and larger area under the receiver operating characteristic curve $(P<0.05, n=4)$. Conclusion: SiSTM imaging is an alternative real-time targeted molecular imaging strategy based on source separation via statistical properties rather than frequency spectra. At conditions relevant to large-artery imaging, SiSTM demonstrated superior contrast, sensitivity, and specificity in vitro when compared with PI + IIR and Harmonic + IIR methods.

\section{$18^{*}$}

Quantitative first-pass perfusion MRI of the mouse heart: data acquisition, reconstruction, and analysis strategies. N.K. Naresh ${ }^{1}, \mathrm{X}$. Chen $^{1}$, P.F. Antkowiak ${ }^{1}$, F.H. Epstein ${ }^{1}$; ${ }^{1}$ University of Virginia, Charlottesville, VA

Experimental therapies for ischemic heart disease, such as stem cells, injectable biomaterials, and gene transfer, are widely investigated in mouse 
models. Also, genetically engineered mice can be used to elucidate the roles of individual genes in myocardial blood flow. First-pass contrast-enhanced MRI is well established in humans. We sought to develop improved quantitative first-pass MRI for mice. Methods: Imaging was performed on a 7-T system (Bruker, Germany). We investigated both dual-bolus and dualcontrast methods. For dual-bolus, a saturation-recovery spiral sequence was employed with two gadolinium $(\mathrm{Gd})$ injections to separately acquire the arterial input and tissue functions (AIF and TF). First-pass images were acquired for one short-axis slice in 5 mice and were analyzed using a Kety model to obtain myocardial $\mathrm{K}_{\text {trans }}$. We also implemented a dual-contrast saturation-recovery Cartesian sequence that employed $\mathrm{k}_{\mathrm{y}}$ and time domain undersampling. For this method, a saturation-recovery gradient-echo sequence acquired two slices within a cardiac cycle, one to obtain the AIF and one to obtain the TF. The AIF slice was acquired with an acceleration factor $=6$ and a $10-\mathrm{ms}$ saturation delay, and the TF slice used an acceleration factor $=4$ and a $70-\mathrm{ms}$ delay. For both slices, the center of k-space was sampled at the Nyquist rate, while higher spatial frequencies were randomly undersampled. Compressed sensing (CS) was used to reconstruct these images. Wild type mice were imaged using $1.25 \%$ isoflurane and were maintained at $37^{\circ} \mathrm{C}$. Results: Using the dual-bolus technique, myocardial $\mathrm{K}_{\text {trans }}$ was $3.3 \pm 1.0 \mathrm{ml} / \mathrm{g} / \mathrm{min}$. Using the dual-contrast technique, the AIF and TF were acquired with a single bolus of $\mathrm{Gd}$ and with acquisition times of $34 \mathrm{~ms} / \mathrm{image}$ for the AIF slice and $50 \mathrm{~ms} / \mathrm{image}$ for the TF slice. The spatial resolution using the dual-contrast sequence was $0.2 \times 0.2 \times 1 \mathrm{~mm}$. Conclusion: First-pass MRI can quantify $\mathrm{K}_{\text {trans }}$ in the mouse heart. Accelerated first-pass MRI using undersampling and CS enables the implementation of a dual-contrast method, which simplifies the procedure (one Gd injection) and may lead to more accurate results because the AIF and TF are acquired under identical physiological conditions. These methods promise to prove useful in the assessment of regenerative and other therapies and may shed light on the roles of individual genes in myocardial perfusion.

\section{9}

Optimized visual assessment of atherosclerotic plaque burden by delayed-time-point FDG-PET/CT imaging: a new observation. $B$. Saboury ${ }^{1}$, B.A. Blomberg ${ }^{1}$, M.H. Gharavi ${ }^{1}$, T.J. Werner ${ }^{1}$, E. Lim² ${ }^{2}$, D. Torigian ${ }^{1}$, G. Cheng ${ }^{2}$, S.R. Akers ${ }^{2}$, A. Alavi1; ${ }^{1}$ Department of Radiology, University of Pennsylvania, Philadelphia, Pennsylvania, ${ }^{2}$ Department of Radiology, Philadelphia VA Medical Center, Philadelphia, Pennsylvania

Our objective was to determine whether PET/CT imaging at delayed time-points following administration of FDG allows for optimal visualization of atherosclerotic plaque in major arteries. Methods: Twenty-one subjects who underwent FDG-PET/CT imaging for preoperative staging of lung cancer were enrolled in this study. Following FDG administration, whole body PET/CT images were acquired at one, two, and three hours. An experienced physician, trained in interpreting PET/CT images, blindly reviewed each study and qualitatively assessed the degree of FDG uptake in the aortic wall using the following rating system: $0=$ no uptake, $1=$ mild uptake, $2=$ moderate uptake, and $3=$ severe uptake. The arterial blood pool served as the reference standard for normal FDG activity. Results: A statistically significant correlation between visual detection of atherosclerotic plaque and the time-point between FDG administration and PET image acquisition was observed Except for 1 subject where no gross change was noted among the three time-points, all other subjects showed statistically significantly improved visualization of aortic atherosclerosis on delayed-time-point images $($ Friedman $=16.22 ;$ Kendall's coefficient $=0.9012 ;$ P-value $=$ 0.0003). Conclusion: Our data demonstrate that standard PET/CT acquisition at 60 minutes after FDG administration is suboptimal for assessment of atherosclerotic plaque. We believe that delayed PET/CT imaging should be adopted as the procedure of choice when FDG-PET/ $\mathrm{CT}$ is employed for detection and quantification of atherosclerotic plaque in the aorta and other major arteries.

\section{0}

In vivo detection of atherosclerotic plaque in $\mathrm{APOE}^{--}$mice using ${ }^{18} \mathrm{~F}$ FDG PET/CT imaging. L. Sleiman ${ }^{1}$, S. Thorn ${ }^{1}$, G. Small ${ }^{1}$, R.A. deKemp ${ }^{1}$, J. DaSilva ${ }^{1}$, R.S. Beanlands ${ }^{1} ;{ }^{1}$ University of Ottawa Heart Institute, Ottawa, Ontario, Canada
F-18-fluorodeoxyglugose $\left({ }^{18} \mathrm{~F}-\mathrm{FDG}\right)$ is a glucose analogue taken up by high-glucose-using cells such as active macrophages found in atherosclerotic lesions. Using ${ }^{18} \mathrm{~F}-\mathrm{FDG}$ as a marker of monocyte metabolism and plaque inflammation, we aimed to determine whether FDG PET could be used to monitor atherogenesis in mice using micro-positron imaging tomography $(\mu \mathrm{PET})$ as well as computed tomography $(\mathrm{CT})$. Methods: Atherogenic $\mathrm{apoE}^{-/-}$mice on a western diet, normally fed adult apoE $\mathrm{E}^{-/-}$mice, and C57BL/6 controls were injected with $1 \mathrm{mCi}{ }^{18} \mathrm{~F}$-FDG followed by imaging with a small-animal PET/CT scanner. The aortas were dissected $2 \mathrm{hrs}$ after tracer injection for ex vivo imaging, autoradiography, and biodistribution studies. Results: Autoradiography demonstrated significant uptake in the aortic lesions corresponding to lesions seen on light microscopy with a correlation coefficient of $r^{2}=0.83$. Aorta ex vivo $\mu$ PET scans show uptake in atherosclerotic lesions as confirmed by light microscopy images. Average SUV for aortic arch of mice on HFD was $0.511 \pm 0.104$ compared to $0.135 \pm$ $0.081(\mathrm{p}=0.001)$ for $\mathrm{apoE}^{-/-}$controls and $0.125 \pm 0.066(\mathrm{p}=0.002)$ for $\mathrm{C} 57 \mathrm{Bl} / 6$ controls. In vivo $\mu \mathrm{PET}$ scans demonstrated higher uptake of ${ }^{18} \mathrm{~F}$ FDG in the atherosclerotic lesions of HFD mice aorta. The average SUV for $\mathrm{ApoE}^{-/-}$on HFD was $4.16 \pm 0.1$ compared to $0.135 \pm 0.08(\mathrm{p}=0.007)$ for $\mathrm{ApoE}^{-/-}$controls and $0.125 \pm 0.066(\mathrm{p}<0.001)$ for $\mathrm{C} 57 \mathrm{bl} / 6$ controls. Biodistribution data illustrate higher ${ }^{18} \mathrm{~F}-\mathrm{FDG}$ uptake in the aorta of mice fed a HFD compared to the control groups. The $\% \mathrm{ID} / \mathrm{g}$ of tissue for the apoE $\mathrm{E}^{-/}$on HFD mice aorta was $11.77 \pm 4.6$ compared to the apoE $\mathrm{E}^{-/}$ controls with $\% \mathrm{ID} / \mathrm{g} 1.26 \pm 0.98(\mathrm{p}=0.008)$ and $2.29 \pm 0.68(\mathrm{p}=0.008)$ for the $\mathrm{C} 57 \mathrm{Bl} / 6$ control mice. Conclusion: ${ }^{18} \mathrm{~F}-\mathrm{FDG}$ is taken up by atherosclerotic plaque in atherogenic $\mathrm{ApoE}^{-/-}$mice while on $\mathrm{HFD}$ as seen by en face autoradiography and is colocalized in plaque as seen on light microscopy. $\mu \mathrm{PET}$ and $\mathrm{CT}$ scan coregistration allow for structural delineation of the $\mu \mathrm{PET}$ images. The use of ${ }^{18} \mathrm{~F}-\mathrm{FDG}$ to image atherosclerosis validates a promising future for use in imaging disease progression and can be extended further to monitor therapy.

\section{1}

F-18 radiolabeling of preformed liposomes for PET imaging of the vascular system. B. Xu ${ }^{1}, \mathrm{H} . \mathrm{Wu}^{1}, \mathrm{~F}$. Bhattacharyya ${ }^{1}, \mathrm{~K} . \mathrm{Lane}^{1}, \mathrm{C} . \mathrm{Li}^{1}, \mathrm{~A}$. Sulima $^{1}$, G.L. Griffiths ${ }^{1}$, E. Jagoda ${ }^{2}$, M. Green ${ }^{2}$, J. Seidel ${ }^{2}$, P. Choyke ${ }^{2}$; ${ }^{1} \mathrm{NHLBI}$, Rockville, MD, ${ }^{2} \mathrm{NCI}$, Bethesda, MD

Nanoscale liposomes have emerged as promising multimodality imaging agents, particularly of the vascular system, due to their extended serum halflives, when pegylated. They also engender significant interest as drug delivery vehicles, for example, doxorubicin liposomes, or Doxil ${ }^{\circledR}$, which is already approved by the FDA for clinical use. In this study, a novel method was developed for the efficient labeling of preformed liposomes, with defined sizes, using copper-free click chemistry. Methods: A (dibenzoyl cyclooctyne) DBCO conjugated PEG lipid was synthesized by conjugating DSPE-PEG2K-Amine with sulfo-DBCO and purified using a reversed phase HPLC method. DBCO functionalized liposomes with a mean diameter between 60 and $70 \mathrm{~nm}$ were made using a lipid mixture consisting of DPPC, cholesterol, DSPE-PEG2K-DBCO, and DSPE-PEG3K at a molar ratio of 58:26:12:4. In trial reactions a fluorescent dye, AlexaFluor-488 azide, was conjugated to DBCO liposomes in PBS over $20 \mathrm{~min}$ at decreasing concentrations of $100 \mu \mathrm{M}, 10 \mu \mathrm{M}, 1 \mu \mathrm{M}, 100 \mathrm{nM}$, and $10 \mathrm{nM}$ of the azide. Once optimum conditions were established, the corresponding radiolabeled hapten (the ${ }^{18} \mathrm{~F}-23$-azido-1-fluoro-3 analog) was prepared and conjugated to the DBCO liposome in similar fashion and purified using a PD10 sizeexclusion column. Micro PET imaging of rats was done at various times following IV injection of the ${ }^{18} \mathrm{~F}$ liposome. Results: An HPLC method for purifying DBCO conjugated lipid and a liposomal formulation for DBCO liposomes were established. Efficient AlexaFluor-488 azide conjugation with preformed DBCO liposome was observed at concentrations as low as $10 \mathrm{nM} .{ }^{18} \mathrm{~F}$ azide was synthesized in a $24 \%(\mathrm{n}=12)$ uncorrected radiochemical yield in $50 \mathrm{~min}$. The conjugation of ${ }^{18} \mathrm{~F}$ azide to $\mathrm{DBCO}$ liposome was done at $37^{\circ} \mathrm{C}$ for $45 \mathrm{~min}$, resulting in an uncorrected radiochemical yield of $47 \%(n=3)$. The overall uncorrected radiochemical yield was $11 \%$ in a $95-$ min synthesis time. Preliminary imaging studies with ${ }^{18} \mathrm{~F}$ liposome appear comparable to images from previously described labeling methods. Conclusion: This is the first time an efficient ${ }^{18} \mathrm{~F}$ radiolabeling of preformed liposomes using copper-free click chemistry has been described, and the new method will allow the ready availability of ${ }^{18} \mathrm{~F}$ liposomes for vascular imaging and targeting. (Funded by the intramural 
program of the National Institutes of Health through the Imaging Probe Development Center and the National Cancer Institute.)

\section{2}

F-18-labeled rhodamines as PET tracers for myocardial perfusion imaging. S. Zhang ${ }^{1}$, M. Bartholomae ${ }^{1}$, S.T. Treves ${ }^{1}$, A.B. Packard ${ }^{1}$; ${ }^{1}$ Children's Hospital Boston/Harvard Medical School, Boston, MA

Our objective was to develop an F-18-labeled radiopharmaceutical for the PET evaluation of myocardial perfusion. Methods: Rhodamine dyes, including rhodamine B (rho-B), rhodamine 123 (rho-123), and rhodamine $6 \mathrm{G}$ (rho-6G), were labeled with F-18 via the F-18 esters. The bistosylates (e.g., ethyl [et], propyl [pr], diethylene glycol [deg], triethylene glycol [teg]) were labeled with F-18 using standard methods, the esters were prepared by reaction of the tosyl fluorides with the rhodamine lactone, and the products were purified by semipreparative HPLC. The F-18-labeled compounds were chemically characterized using the F-19 compounds as reference materials, and their biological properties were measured in vitro (serum stability, uptake by rat cardiac myocytes) and in vivo (biodistribution in normal and infarcted rats). Results: The F-18-labeled esters of rho-B, rho123 , and rho-6G were prepared in $20 \%-40 \%$ decay-corrected yield, high radiochemical purity $(>95 \%)$, and acceptable specific activity. Stability studies revealed that the et ester of rho-B was not stable in mouse serum but was very stable in rat, rabbit, and human serum as well as PBS. Stability studies also showed that the pr, deg, and teg esters of rho-B were significantly more stable in mouse serum than the et ester. This result was confirmed in vivo: Rho-B et ester does not accumulate in the heart in mice, showing high accumulation in the gallbladder, while it does show significant cardiac accumulation in rats. Comparison of the biodistribution of the 4 esters of rho-B showed that deg offered the best combination of stability, cardiac uptake, and liver clearance; thus the deg esters were used for all subsequent studies. Comparison of the cardiac uptake and liver clearance of the F-18-labeled deg esters of rho-B, rho-123, and rho-6G showed that the deg ester of rho-6G had higher cardiac uptake and lower liver uptake/faster liver clearance than the deg esters of rho-B and rho-123. Evaluation of the uptake of the deg ester of rho-6G in a rat myocardial infarct model showed clear infarct delineation, which was confirmed by ex vivo autoradiography and TTC staining. Evaluation of the accumulation of F-18-labeled deg rho$6 \mathrm{G}$ in rat cardiac myocytes showed selective accumulation in the mitochondria that matched that of MitoTracker Green. Conclusion: Fluorine-18-labeled rhodamines, in particular the F-18-labeled deg ester of rho-6G, are promising tracers for the PET evaluation of myocardial perfusion. 